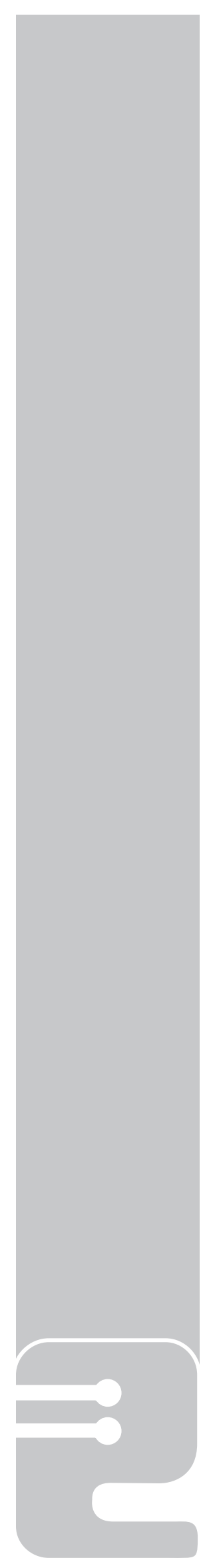

\title{
Propuesta de diseño para un sistema de tratamiento y reutilización de efluentes textiles combinando tecnologías convencionales con el proceso de oxidación avanzada $\left(\mathrm{O}_{3} / \mathrm{H}_{2} \mathrm{O}_{2} / \mathrm{UV}\right)$
}

\author{
Freddy L. Alfonso Moreno ${ }^{1}$, Mónica Z. Choachín ${ }^{2}$, Sara Mendoza Urrea², \\ William E. Pulido Talero ${ }^{3}$
}

Recibido: 24 de febrero de 2015 Aprobado: 23 de mayo de 2015

\section{Resumen}

Se presenta la propuesta de diseño de un sistema de tratamiento de aguas residuales (STAR) aplicado a una industria textil colombiana ubicada en Bogotá, en el que se combinan tecnologías convencionales de tratamiento con tecnologías de oxidación avanzada (TOA). Se genera un sistema de tres etapas: de pretratamiento, que constituye un proceso de cribado, un vertedero de inspección, un tanque de homogeneización y regulación del caudal, y una torre de enfriamiento; de tratamiento primario, que comprende una proceso de coagulación-floculación con posterior clarificación o sedimentación primaria; y de tratamiento terciario o avanzado, para la cual se hace una revisión bibliográfica definiendo el proceso de $\left(\mathrm{O}_{3} / \mathrm{H}_{2} \mathrm{O}_{2} / \mathrm{UV}\right)$ como el óptimo para el tratamiento de colorantes sintéticos complejos utilizados en la industria textil estudiada.

Palabras claves: sistema de tratamiento de aguas residuales (STAR), tecnologías de oxidación avanzada (TOA), colorantes textiles.

\begin{abstract}
The design proposal for a system of wastewater treatment (STAR) applied to a Colombian textile industry located in Bogota, where conventional treatment technologies with advanced oxidation technologies (TOA) combine is presented. 3-stage system is generated: pre-treatment, which constitutes a screening process, a landfill inspection homogenization tank and flow regulation, and a cooling tower; primary treatment stage, forming a coagulation process flocculation with subsequent clarification or primary sedimentation; and a stage of advanced tertiary treatment, for which a bibliographic review is done by defining the process $\left(\mathrm{O}_{3} / \mathrm{H}_{2} \mathrm{O}_{2}\right.$ / UV) as the optimal treatment of complex synthetic dyes used in the textile industry studied.
\end{abstract}

Key words: Advanced oxidation technologies (AOT), waste water treatment system (WWTS), textiles dyes.

\footnotetext{
${ }^{1}$ Químico de la Universidad Nacional de Colombia. M.Sc. en Ingeniería del Instituto Tecnológico de Estudios Superiores de Monterrey, flamoreno17@yahoo.com.ar.

2 Ingeniera ambiental de la Universidad Distrital Francisco José de Caldas, monica.choachi@gmail.com

${ }^{3}$ Ingeniero industrial de la Universidad Distrital Francisco José de Caldas. M.Sc. del Instituto Tecnológico de Estudios Superiores de Monterrey. wpulido@uniminuto.edu
} 


\section{INTRODUCCIÓN}

El sector textil colombiano representa una gran oportunidad de crecimiento y desarrollo socioeconómico para la nación. Constituye un $8 \%$ del PIB (Procolombia, 2014) y es responsable del $15,5 \%$ del empleo industrial, por cuanto se generan más de 450.000 empleos, según Carlos Eduardo Botero Hoyos, presidente del Instituto para la Exportación y la Moda (Inexmoda, 2014).

Dicho sector ha mostrado un incremento en el uso de colorantes complejos sintéticos, de los cuales un amplio número se constituye en colorantes azo o con enlaces $(\mathrm{N}=\mathrm{N})$, que se utilizan para el desarrollo de los procesos de teñido industriales (Adinew, 2012); sin embargo, cerca del $15 \%$ de ellos permanece sin reaccionar y se desechan sin mayor tratamiento a las redes fluviales y de alcantarillado, lo que genera un vertimiento con contaminantes recalcitrantes debido a su naturaleza y conformación química (Apollo, Onyango \& Ochieng, 2014).

Dado lo anterior, dichas industrias tienen la obligación legal y de responsabilidad social de realizar un tratamiento adecuado para estas aguas o efluentes industriales, de manera que no represente un mayor riesgo al equilibrio natural de los ecosistemas que puedan verse afectados.

Las industrias textiles recurren a la implementación de sistemas de tratamiento de aguas residuales (STAR) o plantas de tratamiento de aguas residuales (PTAR), las cuales se basan en una serie de procesos físicos, químicos y biológicos que tienen como finalidad eliminar o disminuir los contaminantes de las aguas servidas industriales o domésticas.

Las tecnologías convencionales usadas para tratar los vertimientos del sector textil se han limitado a mitigar el impacto ambiental, pero son incapaces de realizar un tratamiento que permita la reutilización del recurso en el mismo proceso o en otros. Para la industria textil, los sistemas de tratamiento convencionales constan de pretratamiento, tratamiento primario y en algunas ocasiones tratamiento terciario o avanzado, con el que se busca la decoloración del vertimiento.

Sin embargo la investigación en el tratamiento de efluentes textiles es amplia debido a su complejidad y al gran interés que ha surgido en cuanto al tratamiento de efluentes con tecnologías de oxidación avanzada (TOA), debido a su amplia aplicación y los eficientes resultados que arroja, "se han traducido más de 8000 artículos en revistas de impacto publicados sólo en el periodo 2009-2012". (Sanz, Lombraña \& De Luis, 2012). Estos trabajos ofrecen una nueva visión acerca del desarrollo de estas tecnologías y las impulsa como una tendencia para el tratamiento y mineralización de colorantes sintéticos complejos.
Las tecnologías de oxidación avanzada se fundamentan en procesos de oxidación química en fase acuosa para contaminantes, en los que se involucra la generación de especies hidroxilo - $\mathrm{OH}$ altamente oxidantes e inestables que reaccionan rápidamente y pueden degradar con facilidad dichos contaminantes (Glaze, 1987). El objetivo de estos procesos es mineralizar los contaminantes degradándolos a sus formas más simples en $\mathrm{CO}_{2}$, $\mathrm{H}_{2} \mathrm{O}$ y ácidos como $\mathrm{HCl}$; la reacción general estaría representada como se muestra a continuación (Osorio, Torres \& Sánchez, 2011).

$\mathrm{POA} \rightarrow \mathrm{OH}^{-} \overrightarrow{\text { contaminante }} \mathrm{CO}_{2}+\mathrm{H}_{2} \mathrm{O}+$ ionesinorgánicos

El proyecto se lleva a cabo en la ciudad de Bogotá (Colombia), por lo cual se toman los estándares exigidos por la autoridad ambiental competente, para este caso la Secretaría de Ambiente, que en su condición de ente regulador expide la Resolución 3957 de 2009, norma técnica para el control y manejo de los vertimientos realizados a la red de alcantarillado público en el Distrito Capital. Además, se fijan los índices, factores, concentraciones o estándares máximos para su vertido y se establece el formulario de solicitud de registro de vertimientos y se toman algunos referentes del diseño hidráulico de las directrices dadas por el Reglamento técnico del sector de agua potable y saneamiento básico (RAS, 2000).

\section{METODOLOGÍA}

El proyecto empieza con la recopilación de los requisitos legales, técnicos y de toda la documentación en materia de tecnologías convencionales y tecnologías emergentes asociadas al sector textil. Se desarrolla en las condiciones reales de una industria textil localizada en la zona industrial del barrio Carvajal, en Bogotá, en la cual se lleva a cabo un proceso productivo de tipos $B$ y $C$ según MADS (2003).

Esta investigación se desarrolló en cuatro etapas. En la primera, calidad del vertimiento, se realizaron pruebas de calidad de agua en una muestra compuesta, con los parámetros requeridos en la normativa vigente en el Distrito Capital para el 2014, (Resolución 3957 de 2009), y dos muestras específicas tomadas al finalizar el proceso unitario de teñido para colores considerados críticos en la industria en cuestión; en estos últimos muestreos se evaluaron los parámetros de calidad de agua DBO, DQO y color, que son los más representativos para el sector textil.

En la segunda etapa, orientación de concepto, se busca determinar los lineamientos generales de un sistema de tratamiento de aguas industriales para el sector textil, 
en el cual se fijan los parámetros de incumplimiento de normativa, de riesgo por incremento en producción, y los requeridos para una posible reutilización dentro del recurso en el proceso productivo.

En la tercera etapa, orientación estratégica, se definen los procesos unitarios, y se desarrolla un diagrama de procesos del sistema de tratamiento con el propósito de desarrollar, en la etapa final de diseño específico, los cálculos hidráulicos y otros correspondientes.

\section{RESULTADOS}

\subsection{Calidad del vertimiento}

Se comparan los resultados del análisis fisicoquímico del vertimiento con lo exigido por la normativa aplicable, en este caso la Resolución 3957 de 2009. Los hallazgos se encuentran en la tabla 1 y las comparaciones de las muestras específicas en las tablas 2 y 3 .

\begin{tabular}{|c|c|c|c|c|}
\hline PARÁMETRO & UNIDAD & $\begin{array}{l}\text { RESULTADO DEL } \\
\text { ANÁLISIS }\end{array}$ & $\begin{array}{l}\text { LÍMITE NORMATIVO } \\
\text { (Resolución } 3957 \text { de 2009) }\end{array}$ & CUMPLIMIENTO \\
\hline Dbo (5) & $\mathrm{mg} / \mathrm{L}$ & 462,0 & 800 & sí \\
\hline Dqo & $\mathrm{mg} / \mathrm{L}$ & 951,4 & 1500 & sí \\
\hline Color & Unidades Pt - Co & 8000 & 50 u. en dilución $1 / 20$ & NO \\
\hline $\mathrm{Ph}$ & Unidades & 5,42 & $5.0-9.0$ & Sí \\
\hline Sólidos sedimentables & $\mathrm{mg} / \mathrm{L}$ & $<0,1$ & 2 & Sí \\
\hline Sólidos suspendidos totales & $\mathrm{mg} / \mathrm{L}$ & 134,5 & 600 & Sí \\
\hline Temperatura & ${ }^{\circ} \mathrm{C}$ & 22,1 & 30 & Sí \\
\hline Tensoactivos (SAAM) & $\mathrm{mg} / \mathrm{L}$ & 1,54 & 10 & sí \\
\hline Grasas y aceites & $\mathrm{mg} / \mathrm{L}$ & 74,5 & 100 & Sí \\
\hline Fenoles & $\mathrm{mg} / \mathrm{L}$ & 0,60 & 0,2 & NO \\
\hline Plomo total & $\mathrm{mg} / \mathrm{L}$ & $<0,40$ & 0,1 & sí \\
\hline Cadmio total & $\mathrm{mg} / \mathrm{L}$ & $<0,04$ & 0,02 & Sí \\
\hline Cromo total & $\mathrm{mg} / \mathrm{L}$ & $<0,20$ & 1 & Sí \\
\hline Cobre total & $\mathrm{mg} / \mathrm{L}$ & $<0,10$ & 0,25 & Sí \\
\hline Sulfuros totales & $\mathrm{mg} / \mathrm{L}$ & 4,3 & 5 & Sí \\
\hline
\end{tabular}

\begin{tabular}{|c|c|c|c|}
\hline \multicolumn{2}{|c|}{ Tabla 2. Comparación de la caracterización del vertimiento puntual (colorante negro) con la exigencia normativa. } \\
\hline UNIDAD & RESULTADO DEL ANÁLISIS & $\begin{array}{c}\text { LímITE NORMATIVO } \\
\text { (Res. 3957/09) }\end{array}$ & CUMPLIMIENTO \\
\hline $\mathrm{mg} / \mathrm{L}$ & 1344 & 800 & NO \\
\hline $\mathrm{mg} / \mathrm{L}$ & 5020 & 1500 & NO \\
\hline Unidades Pt - Co & 12.000 & 50 u. en dilución 1/20 & NO \\
\hline \multicolumn{2}{|r|}{ Fuente: Quimicontrol (2015), modificado por los autores. } \\
\hline
\end{tabular}

\begin{tabular}{|c|c|c|c|}
\hline \multicolumn{3}{|c|}{ Tabla 3. Comparación de la caracterización del vertimiento puntual (colorante naranja) con la exigencia normativa. } \\
\hline UNIDAD & RESULTADO DEL ANÁLISIS & $\begin{array}{c}\text { LíMITE NORMATIVO } \\
\text { (Resolución 3957 de 2009) }\end{array}$ & CUMPLIMIENTO \\
\hline DBO (5) & $\mathrm{mg} / \mathrm{L}$ & 954 & 800 \\
\hline DQO & $\mathrm{mg} / \mathrm{L}$ & 2003 & 1500 \\
\hline Color & Unidades Pt - Co & 7000 & 50 u. en dilución 1/20 \\
\hline \multicolumn{2}{|r|}{ Fuente: Quimicontrol (2015), modificado por los autores. } \\
\hline
\end{tabular}




\subsection{Orientación de concepto}

Según los resultados presentados en la tabla 1 , los parámetros que incumplen con los límites permisibles por la SDA son color y fenoles; sin embargo, cuando se realiza el muestreo puntual (tablas 2 y 3 ) se evidencia incumplimiento en todos los parámetros analizados DBO, DQO y color. Esto indica que si la empresa llega a aumentar su capacidad en el proceso de teñido, que es el que genera más impacto en la calidad del vertimiento, su carga contaminante aumentará y probablemente incumplirá con más de dos parámetros, lo que obliga a generar estrategias para el tratamiento de sus vertimientos.

Dado lo anterior, se diseñó la STAR enfocada en la disminución de parámetros como color, fenoles, demanda química y biológica de oxígeno, temperatura y tensoactivos, los cuales se reportan en los análisis como los que incumplen la normativa o están cercanos al límite para incumplimiento y representan un riesgo en la calidad. El color cumple un papel principal ya que se considera que los colorantes son los que influyen sobre la mayoría de variables, al evidenciar una carga contaminante muy superior cuando se realiza la muestra con un color negro.

Para determinar si en el sistema de tratamiento debe implementarse o no un tratamiento secundario, se calculó el indicador de biodegradabilidad, que permite identificar la capacidad del vertimiento para transformarse en una estructura química más simple por vía microbiana. Se determina mediante la relación de la demanda biológica y la demanda química de oxígeno, como se muestra en la (Ec. 1), y finalmente se clasifica el vertimiento según el nivel de biodegradabilidad, como lo proponen Ardila, Reyes \& Arriola (2009). Los rangos se evidencian en la tabla 4.

$$
B=\frac{D B O s}{B Q O} \quad(E c .1)
$$

\begin{tabular}{|c|c|}
\hline \multicolumn{2}{|c|}{ Tabla 4. Criterios de biodegradabilidad según la relación $\mathrm{DBO}_{5} / \mathrm{DQO}$} \\
\hline $\mathrm{DBO5} / \mathrm{DQO}$ & CARÁCTER \\
\hline$>0,8$ & Muy biodegradable \\
\hline $0,7-0,8$ & Biodegradable \\
\hline $0,3-0,7$ & Poco biodegradable \\
\hline$>0,3$ & No biodegradable \\
\hline \multicolumn{2}{|c|}{ Fuente: Ardila, Reyes \& Arriola (2009) } \\
\hline
\end{tabular}

Desarrollando, se obtiene la tabla 5.

\begin{tabular}{|c|c|}
\hline \multicolumn{2}{|c|}{ Tabla 5. Resultados de biodegradabilidad del efluente. } \\
\hline MUESTRA & DBO5/DQO \\
\hline 1 & 0,485600168 \\
\hline 2 & 0,494318182 \\
\hline 3 & 0,476285572 \\
\hline Promedio & 0,485401307 \\
\hline
\end{tabular}

Se evidencia que tanto los resultados de cada muestra como el valor promedio se encuentran en el rango de $0,3-0,7$, lo que indica que el vertimiento presenta características poco biodegradables, y que un tratamiento por vía microbiana va a resultar con eficiencias muy bajas. Dado lo anterior, se decidió suprimir la fase del tratamiento secundario del STAR y se descartó cualquier tipo de tratamiento terciario basado en degradación por medio de microorganismos.
La factibilidad de reutilización de los vertimientos en el mismo proceso productivo condujo a la revisión de bibliografía concerniente a este tema. Debido a que en la normativa colombiana no se determinan los parámetros para la reutilización del agua a escala industrial, se tomó la propuesta de la regulación española para dicho aspecto (Real Decreto 1620 de 2007), el cual limita en variables biológicas, que no se miden en esta investigación, y las variables de sólidos suspendidos totales con un límite de $35 \mathrm{mg} / \mathrm{L}$. 


\subsection{Orientación estratégica}

Los procesos correspondientes al pretratamiento, tales como cribado o tamizado, torre de enfriamiento y tanque de homogeneización/neutralización son necesarios y casi obligatorios para cualquier sistema de tratamiento de aguas residuales en la industria textil.

Es necesario implementar un tratamiento primario, en el que se toma el proceso de coagulación/floculación en complemento con el tanque de sedimentación. Hasta este punto el sistema podría presentar una eficiencia alta en varios parámetros; sin embargo, dado que la industria utiliza colorantes de tipo disperso y reactivo, no se garantiza que el tratamiento primario sea exitoso ya que, según Colindres Bonilla (2010), el proceso de floculación/coagulación es efectivo en colorantes sulfurosos y dispersos debido a que son capaces de formar conglomerados de gran tamaño, haciendo la remoción posible. Lo anterior implica realizar el conteo de los colorantes empleados en la industria analizada, en lo cual se evidencia que tan sólo el $27 \%$ de los colorantes empleados son de tipo disperso, lo que establece que el parámetro color puede tener eficiencias bajas en el proceso.

Dado lo anterior, se requiere implementar un proceso de tratamiento terciario en el STAR, referido al uso de las tecnologías de oxidación avanzada, que comprenden una gran variedad de procesos con diversas ventajas y desventajas, calificadas a escala mundial como una tecnología apta para degradar compuestos orgánicos complejos como los colorantes empleados en la industria textilera.

Existen cerca de 18 procesos de oxidación avanzada, aplicando en este caso el de $\mathrm{UV} / \mathrm{H}_{2} \mathrm{O}_{2} / \mathrm{O}_{3}$, que reporta altas eficiencias y el tiempo de exposición es corto, según lo menciona Yonar (2009) al encontrar que este POA es más eficiente en términos de decoloración para efluentes provenientes de la industria textil dado que arroja valores superiores al $90 \%$. Se estudian otros procesos de gran aplicabilidad como $\left(\mathrm{O}_{3} / \mathrm{H}_{2} \mathrm{O}_{2}\right),\left(\mathrm{O}_{3} / \mathrm{UV}\right)$ y $\left(\mathrm{H}_{2} \mathrm{O}_{2} / U V\right)$. Sin embargo, según Shu \& Chang (2005), se encontró que el proceso de oxidación $\left(\mathrm{H}_{2} \mathrm{O}_{2} / \mathrm{UV}\right)$ necesitaba más tiempo para eliminar el color que el proceso $\left(\mathrm{UV} / \mathrm{O}_{3}\right)$, lo cual generó una degradación del carbono orgánico total con eficiencias del $99 \%$ y 33 $\%$, respectivamente, para un tiempo de exposición de 160 minutos. De esta forma, la sinergia entre estos dos procesos $\left(\mathrm{O}_{3} / \mathrm{H}_{2} \mathrm{O}_{2} / \mathrm{UV}\right)$ para la eliminación de contaminantes orgánicos coloreados reducirá el tiempo de degradación del color, mantendrá la eficiencia y disminuirá el carbono orgánico total. La figura 1 muestra los procesos y operaciones unitarias que formarán parte del STAR propuesto.

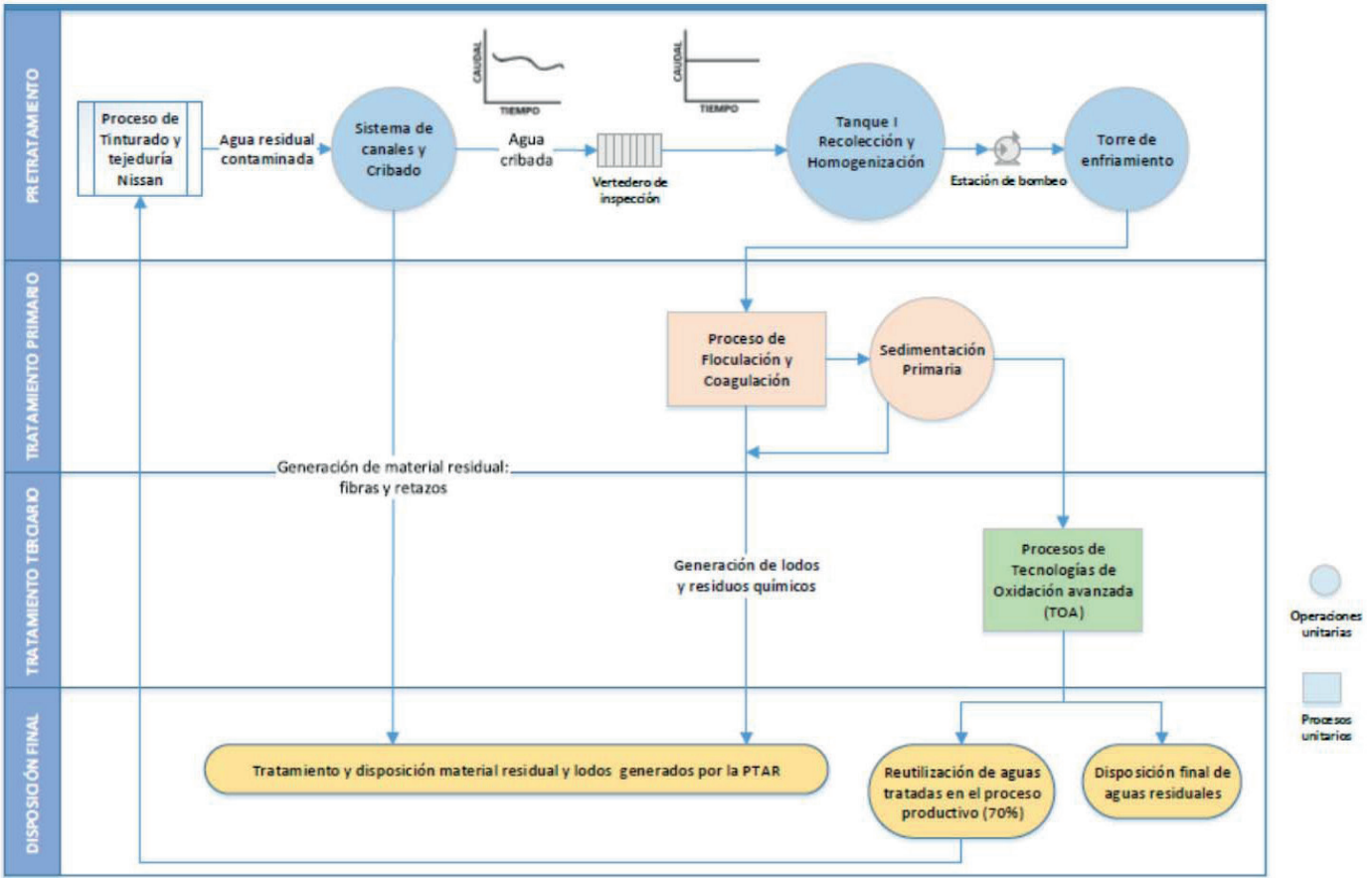

Figura 1. Diagrama de flujo de los procesos y operaciones unitarias por realizar en el STAR. Fuente: autores (2015). 


\subsection{Diseño específico}

En la tabla 6 se definen los caudales de diseño anteriormente calculados para la industria específica escogida.

\begin{tabular}{|c|c|c|}
\hline \multicolumn{3}{|c|}{ Tabla 6. Resultados del caudal de diseño. } \\
\hline DESCRIPCIÓN & $(\mathrm{l} / \mathrm{sg})$ & $\mathrm{m}^{3} / \mathrm{s}$ \\
\hline Caudal máximo & 9,34500 & 0,00935 \\
\hline Caudal promedio & 2,20033 & 0,00220 \\
\hline Caudal mínimo & 0,14160 & 0,00014 \\
\hline \multicolumn{2}{|c|}{ Fuente: elaboración propia } \\
\hline
\end{tabular}

Para la fase del pretratamiento se toma el caudal máximo de 9,34 l/ s, de manera que no haya riesgo de sobrecarga del sistema. La estructura y sus dimensiones se pueden observar en la figura 2.



En el numeral 9 de la figura 2 se observa el sistema de refrigeración del efluente, constituido por una torre de enfriamiento que no fue diseñada por los autores pero reúne las características necesarias para el correcto funcionamiento y acoplamiento con el STAR.
Para el dimensionamiento del tratamiento primario se tuvo en cuenta el test de jarras realizado con anterioridad para el vertimiento escogido. Así, se tienen en la tabla 7 los coagulantes, los floculantes y las dosis óptimas para un correcto tratamiento.

Tabla 7. Ensayo de tratabilidad. Test de jarras.

Compuestos químicos utilizados: como coagulantes se utilizó cloruro férrico, hidroxicloruro de aluminio, cal, sulfato de aluminio grados A y B, hidróxido de sodio, cloruro de calcio y algunos polielectrolitos. Se obtuvieron buenos resultados con hidroxicloruro y polielectrolito Cyquim superfloc A-130.

Compuestos químicos seleccionados: se seleccionó como coagulante hidroxicloruro y polielectrolitoCyquimsuper flocA-130.

\begin{tabular}{|c|c|c|}
\hline \multicolumn{3}{|c|}{ CONDICIONES DEL ENSAYO } \\
\hline COMPUESTOS QUÍMICOS & UNIDAD & $\begin{array}{l}\text { DÓSIS } \\
\text { ÓPTIMA }\end{array}$ \\
\hline Hidroxicloruro de aluminio al $10 \%$ & $\mathrm{mg} / \mathrm{L}$ & 6 \\
\hline Polielectrolito Cyquim superfloc A-130 & $\mathrm{mg} / \mathrm{L}$ & 1 \\
\hline Tiempo de mezcla rápida & minutos & 0,5 \\
\hline Tiempo de mezcla lenta & minutos & 10 \\
\hline Gradiente de mezcla lenta & $\operatorname{seg}^{-1}$ & 30 \\
\hline Tiempo de sedimentación & minutos & 10 \\
\hline
\end{tabular}


El esquema del tratamiento primario puede observarse en la figura 3, en la que se evidencia que los tanques de mezcla rápida y lenta funcionan en una misma estructura, de tal manera que se aprovecha el espacio con el que cuenta la industria escogida.

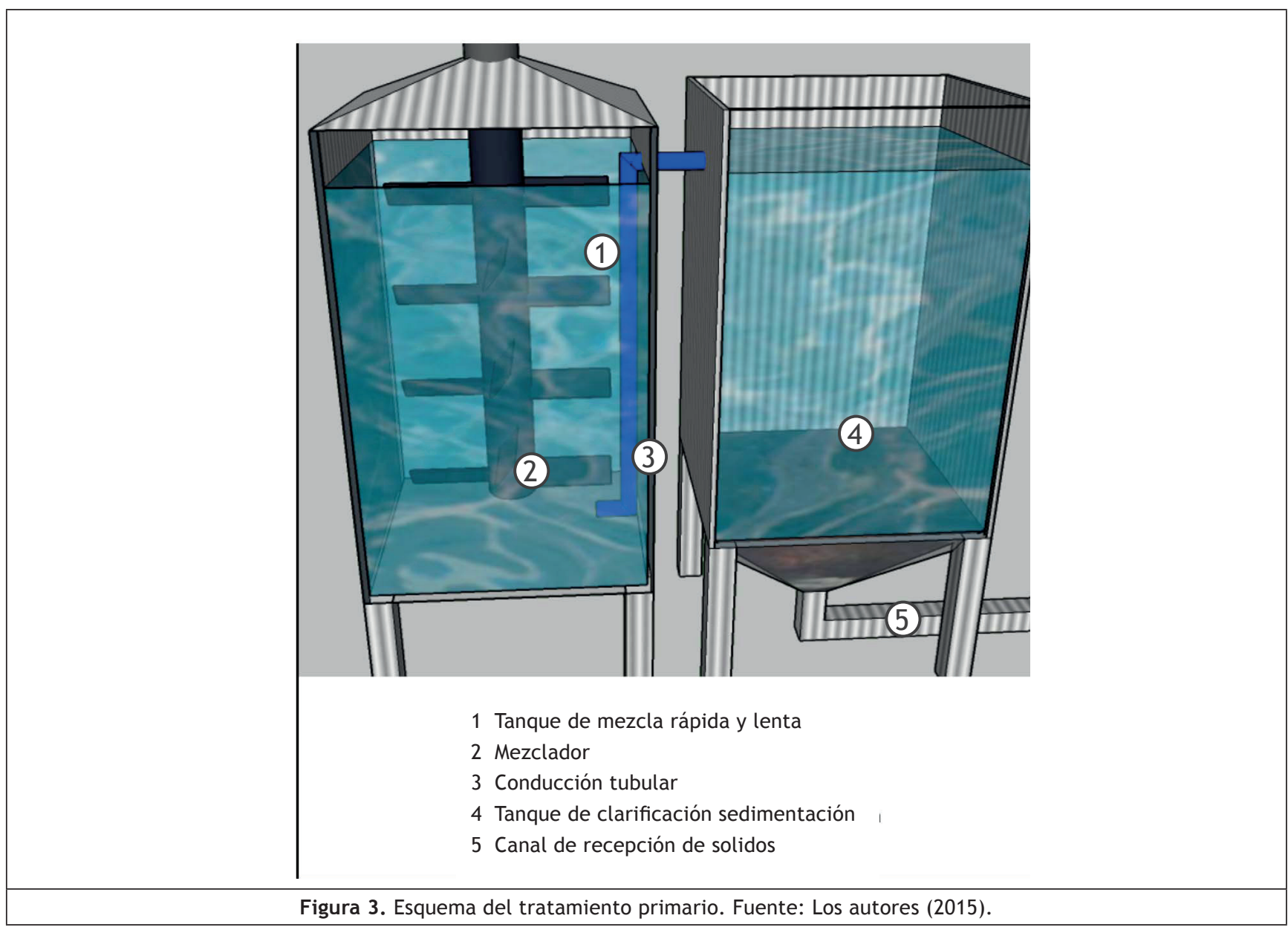

Para el tratamiento terciario o avanzado se realiza un diseño netamente teórico basado en información bibliográfica revisada con estudios previos, con pruebas en plantas pilotos, entre otros.

En la tabla 8 se presentan las dosis y concentraciones óptimas teóricas para el funcionamiento del proceso $\mathrm{O}_{3} / \mathrm{H}_{2} \mathrm{O}_{2} / \mathrm{UV}$.

\begin{tabular}{|c|c|c|c|}
\hline \multicolumn{5}{|c|}{ Tabla 8. Valores específicos de los agentes oxidantes del POA. } \\
\hline OXIDANTE & VALOR & UNIDAD & OBSERVACIÓN \\
\hline UV & 240 & $\mathrm{~nm}$ & Longitud de onda \\
\hline $\mathrm{O}_{3}$ & 18,4 & $\mathrm{mg} / \mathrm{C}$ & Caudal \\
\hline $\mathrm{H}_{2} \mathrm{O}_{2}$ & 1 & $\%$ & Concentración \\
\hline \multicolumn{4}{|c|}{ Fuente: autores (2015). } \\
\hline
\end{tabular}

Se tomaron quince minutos como tiempo de exposición a agentes oxidantes, por lo cual se buscó desarrollar un sistema con alta capacidad de retención, bajas velocidades y uso eficiente del espacio. Se adaptó el diseño de un floculador hidráulico de flujo horizontal y los cálculos se efectuaron de acuerdo con las exigencias hidráulicas del mismo; como resultado se aprecia el esquema en la figura 4. 


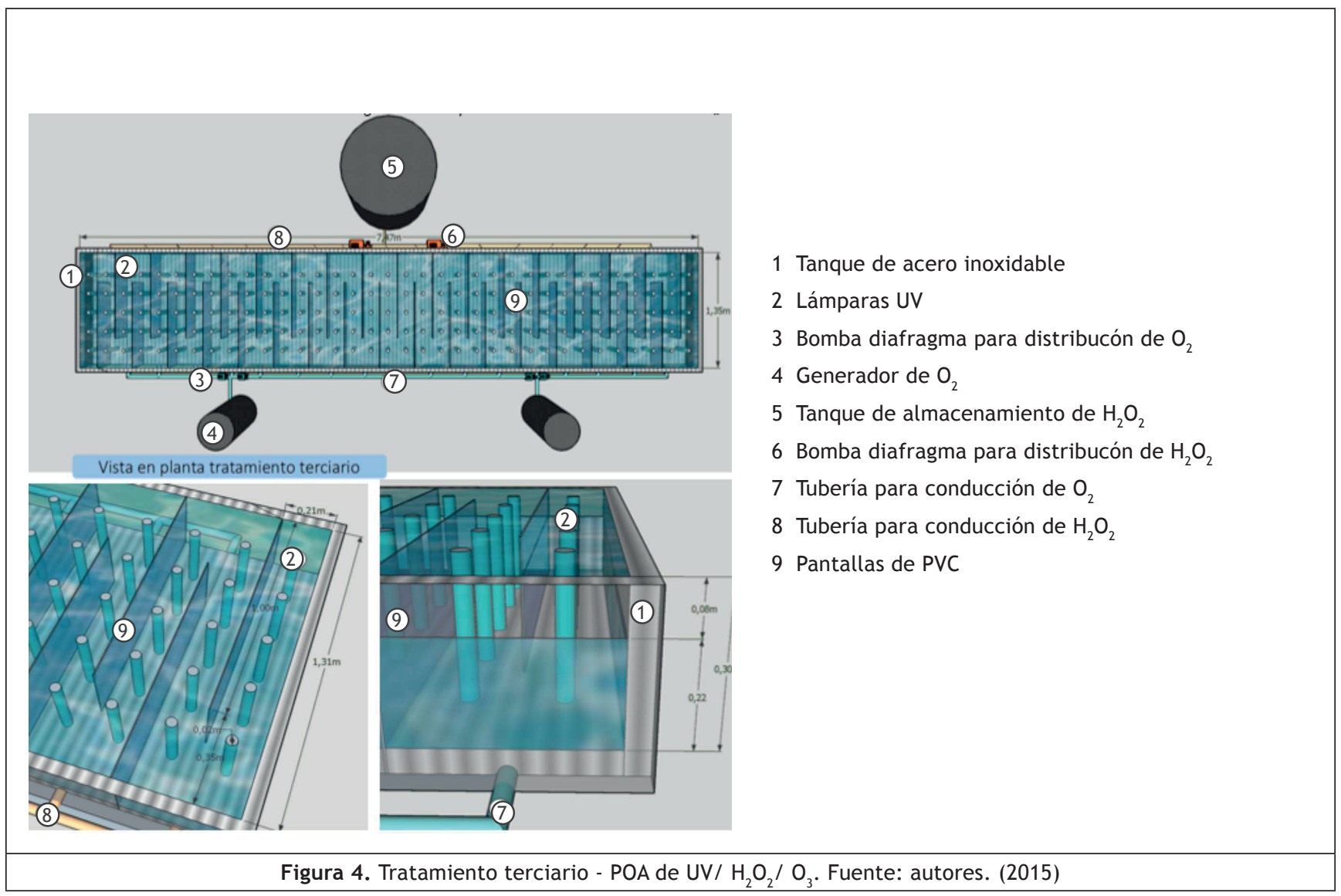

\section{CONCLUSIONES}

Con el tratamiento propuesto se genera un efluente que cumple con los requerimientos ambientales exigidos por la legislación colombiana, teniendo en cuenta que se trabajó en condiciones específicas en la industria escogida y sabiendo que, en general, la industria textil se caracteriza por la generación de grandes concentraciones de DBO5, DQO y COT.

Dado que el diseño y dimensionamiento del tratamiento terciario se basó en la teoría encontrada, es imperativo implementar un prototipo en el que se puedan estudiar las mejores condiciones de los agentes oxidantes, de manera que haya certeza en el funcionamiento y eficiencia del proceso de $\mathrm{UV} / \mathrm{H}_{2} \mathrm{O}_{2} / \mathrm{O}_{3}$ escogido.

Dentro de las tecnologías emergentes valoradas se han catalogado los procesos de oxidación avanzada como una tecnología ambiental y limpia capaz de mineralizar una gran variedad de compuestos que representan un riesgo de contaminación, con resultados de alta efectividad en la degradación de colorantes y eficiencia del $99 \%$, lo que ha despertado el interés investigativo de las industrias textiles para que sean una alternativa de implementación dentro de sus sistemas de tratamientos de vertimientos industriales.
El dimensionamiento hidráulico del sistema de tratamiento se desarrolló para cada uno de los procesos unitarios definidos, teniendo en cuenta las condiciones específicas identificadas en la industria escogida, percibiendo como limitante la inexistencia de una norma técnica que regule los criterios para el diseño de plantas de tratamiento a escala industrial, por lo que se adaptaron directrices del RAS 2000, apropiado para aguas residuales domésticas.

\section{REFERENCIAS}

1. Adinew, B. (2012). Textiles effluent treatment and decolorization techniques - A review. Chemistry: Bulgarian Journal of Science Education, pp. 434-456.

2. Apollo, S., Onyango, M. \& Ochieng, A. (2014). Integrated UV photodegradation and anaerobic digestion of textile dye for efficient biogas production usind zeolite. Chequical Engineering Journal, pp. 241-247.

3. Ardila, A. N., Reyes, J. \& Arriola, E. (2012). Degradacion fotocatalítica de materia orgánica no biodegradable presente en efluentes de la industria farmacéutica. Revista Politécnica, 
8(15). Medellín: Politécnico Colombiano Jaime Isaza Cadavid.

4. Glaze, W. (1987). Drinking-water treatment with ozone. Environmental Science Technology, (21), pp. 224-230.

5. Inexmoda. (4 de diciembre de 2014). Inexmoda. Recuperado de http://prensa.inexmoda.org.co/ news/233/colombiatex-de-las-amricas--epicentrode-negocios-del-sector-textil--confeccin.html.

6. MADS. (2003). Guía de buenas prácticas para el sector textiles. Bogotá, Colombia: Presidencia de la República - Fundes. Recuperado de MADS: http://www.minambiente.gov.co/documentos/ Textiles.pdf.

7. Osorio, F., Torres, J. \& Sánchez, M. (2011). Tratamiento de aguas para la eliminación de microorganismos y agentes contaminantes: aplicación de procesos industriales a la reutilización de aguas residuales. Zaragoza: Ediciones Díaz de Santos.

8. Perkowski, J. \& Kos, L. (2003). Decolouration of model dye house wastewater with advanced oxidation processes. Fibres and textiles in Eastern Europe, 11, pp. 67-71.
9. Procolombia. (2014). Procolombia: exportaciones, turismo, inversión, marca, país. Recuperado de http: / / www. inviertaencolombia. com.co/sectores/manufacturas/textil-yconfeccion.html.

10. Resolución 3957 de la Secretaría Distrital de Ambiente, junio de 2009. Recuperado de http: / / www.alcaldiabogota.gov.co/sisjur/normas/ Norma1.jsp?i=37051.

11. Sanz, J., Lombraña, J. I. \& de Luis, A. (2012). Estado del arte en la oxidación avanzada a efluentes industriales: nuevos desarrollos y futuras tendencias. España: Departamento de Ingeniería Química y del Medio Ambiente UPV/EHU.

12. Shu, H. \& Chang, M. (2005). Pre-ozonation coupled whit UV/ $\mathrm{H}_{2} \mathrm{O}_{2}$ process for the decolorization and mineralization of cotton dyeninf effluent and synthesized C.I Direct Black 22 wastewater. Journal of Hazardous Materials, pp. 127-133.

13. Yonar, T. (2009). Decolorisation of textile dyeing effluents using advanced oxidation processes. Turkey: Uludag University, Enviromental Engineering Department. 